\title{
Union libre ou mariage forcé?
}

\author{
Laura Bertini dans l'interview avec Stefan Spring (StS)
}

De nouvelles technologies sont mises au service de l'allégement et de la sécurité du quotidien. Mais quelque fois les choses se passent autrement que voulu. Laura Bertini a analysé l'achoppement d'un projet en Suisse italienne.

\section{Lebensgemeinschaft oder Zwangsehe?}

Es werden neue Technologien angeboten, um den Alltag zu erleichtern und sicherer zu machen. Jedoch entwickeln sind die Dinge manchmal anders als beabsichtigt. Laura Bertini hat die Hindernisse im Zusammenhang mit einem Projekt in der italienischsprachigen Schweiz analysiert.

DOI 10.1024/2297-5160/a000051

StS : La SUPSI a développé une série de propositions techniques pour augmenter la sécurité des personnes dans leur habitation. Quels étaient les objectifs du projet IDA-GRANNO et comment la SUPSI construit ses démarches?

Laura Bertini : IDA-GRANNO a été un projet fortement interdisciplinaire et complexe. L'équipe de travail visait le soutien de l'autonomie à domicile et un renforcement du sentiment de sécurité à travers un bouquet technologique à caractère sanitaire adaptable à l'usager. Par le biais d'une programmation des objets de la vie quotidienne le système permettait d'automatiser certaines tâches du quotidien ou en sécuriser d'autres. La fonctionnalité de détection des chutes, une tâche très compliquée à développer, a constitué le moteur du projet et en même temps un défis qui s'est révélé trop ambitieux. Le but était de développer, au sein du bouquet, une montre capable de détecter les chutes et alerter de façon passive les parents ou les services de soins, ceci pour diminuer les conséquences de cet événement associé de façon particulière à l'avancé en âge.

StS : Les chutes sont donc identifiées comme origine de coûts et de dépendance. Vous critiquez ce choix, pourquoi?

L'épidémiologie a défini l'âge comme facteur de risque pour l'ostéoporose. Dès lors la chute a émergé comme thématique de santé publique. Il s'agit d'un référentiel commun, lorsque « la chute chez la personne âgée » est évoquée, il y a un consensus général autour de l'idée qu'il s'agit d'un événement déclencheur de toute une série de problématiques, dont la perte d'autonomie. Pourtant il est très difficile de définir la chute de façon univoque. Avec ce terme on désigne une multitude de situations fort différentes entre elles, c'est pour cela qu'il faut faire très attention à se référer à la chute lorsqu'on travaille à des solutions socio-techniques. Les personnes ne tombent pas de la même façon et surtout elles ne donnent pas la même interprétation à toutes les chutes. La chute a aussi des fonctions symboliques, elle est un marqueur de l'entrée dans la vieillesse renvoyant à une perte de maitrise. La peur de tomber, qui est un facteur de risque elle aussi, a été définie par les personnes âgées fragiles enrôlées dans de tels projets, comme étant la peur de ne pas pouvoir se relever. Alors, la problématique s'inverse et la solution socio-technique ne peut plus viser la détection d'un corps qui tombe associé à une alerte automatisée et indépendante de l'usager qui rassurait beaucoup les proches aidants. Finalement, si on ne tombe pas comme l'on fait les jeunes personnes pendant les tests de laboratoire se jetant sur un matelas, alors l'alerte pourrait ne pas se déclencher. Les personnes lorsqu'elles sentent le besoin de se mettre en sécurité, souhaitent pouvoir déclencher une alerte de façon autonome et simple, car l'enjeux n'est pas simplement un corps qui tombe. Lorsqu'on se place du côté de l'usager et on quitte le point de vue de la prise en charge médicale, les besoins qui doivent être traduit techniquement changent la façon de concevoir les objets et leurs fonctionnalités.

StS : La personne âgée se trouve donc confrontée à des « engins » de prévention pour des problèmes qu'elle ne ressent pas les siens. La technologie présentée comme « sauveur » perd dans ses yeux tout intérêt. En partant de ce projet, quelles seraient vos remarques critiques sur l'introduction de supports techniques au niveau du soutien de la personne âgée et des soins qu'elle éventuellement demande?

Les personnes, âgées ou pas, lorsqu'elles sont confrontées à la difficulté d'accomplir une tâche de la vie quotidienne, développent différentes stratégies de compensation. L'environnement de vie n'est pas seulement un espace objectif, il est surtout un espace de repères subjectifs de notre être au monde. Si on introduit un changement tel qu'un dispositif technique, celui-ci peut soit nous rendre plus efficace pour cette tâche, soit nous rendre étranger à notre environnement par un effet de déstructuration. Par exemple, un distributeur de médicaments était programmé pour alerter la personne (par un son) et ensuite les proches aidants en cas d'oubli de la prise de médicaments à l'heure établie. L'usager, qui auparavant prenait la thérapie en accord avec le signal sonore du radiojournal de midi, a ensuite utilisé l'alerte de l'appareil pour procéder à la prise. L'usage prévu, c'est-à-dire un contrôle de la prise et une intervention 
en cas d'oubli, n'était donc pas très compatible avec l'intérêt de la personne à ne pas oublier sa thérapie.

StS : L'hétérogénéité des personnes âgées et les aspects subjectifs de la vie sont probablement les aspects le plus souvent oubliés lorsqu'on part d'un point de départ technologique pour chercher des réponses aux aspects problématiques du nombre croissant de personnes âgées dans la société. N'y-a-t-il donc pas d'espace pour des apports techniques dans notre domaine?

Les personnes âgées, comme toutes personnes, font preuve d'une grande capacité d'inventivité et d'adaptation aux conditions, parfois difficiles, de la vie quotidienne. Aînés ou pas, les usagers d'un objet technique s'approprient des dispositifs lorsqu'ils jugent que celui-ci leur permet de s'approprier subjectivement ou objectivement un peu plus du monde que les entourer. En d'autres termes, nous aimons être plus efficace lorsqu'on adopte un outil quelconque, autrement on l'abandonne aussi vite. Toute solution technique doit partir d'un besoin clairement définit par l'usager et répondre à ses nécessités en termes d'usabilités, c'est-à-dire que le dispositif doit être maîtrisable par la personne en fonction de son propre corps et son environnement et la façon dont elle les habite.

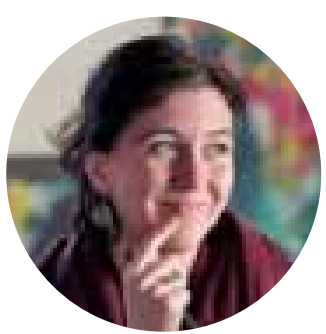

Dr. Laura Bertini, est anthropologue et chercheuse au Centro competenze anziani de la Haute école spécialisée de la Suisse italienne.

laura.bertini@supsi.ch

Stefan Spring est chargé scientifique à l'Union centrale suisse pour le bien des aveugles (UCBA).

spring@szb.ch

\section{Déjà oublié ce que vous venez de lire?}

\section{Alzheimer ou une autre forme de démence peut toucher tout le monde.

